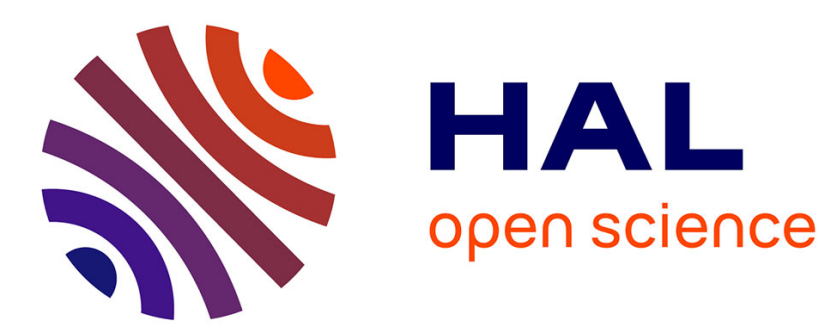

\title{
Dynamic decision making with feasibility goals: A procedural-rationality approach
}

Johannes Binswanger

\section{To cite this version:}

Johannes Binswanger. Dynamic decision making with feasibility goals: A proceduralrationality approach. Journal of Economic Behavior and Organization, 2011, 78 (3), pp.219. 10.1016/j.jebo.2011.01.006 . hal-00989522

\section{HAL Id: hal-00989522}

\section{https://hal.science/hal-00989522}

Submitted on 12 May 2014

HAL is a multi-disciplinary open access archive for the deposit and dissemination of scientific research documents, whether they are published or not. The documents may come from teaching and research institutions in France or abroad, or from public or private research centers.
L'archive ouverte pluridisciplinaire HAL, est destinée au dépôt et à la diffusion de documents scientifiques de niveau recherche, publiés ou non, émanant des établissements d'enseignement et de recherche français ou étrangers, des laboratoires publics ou privés. 


\section{Accepted Manuscript}

Title: Dynamic decision making with feasibility goals: A procedural-rationality approach

Author: Johannes Binswanger

PII: $\quad$ S0167-2681(11)00020-5

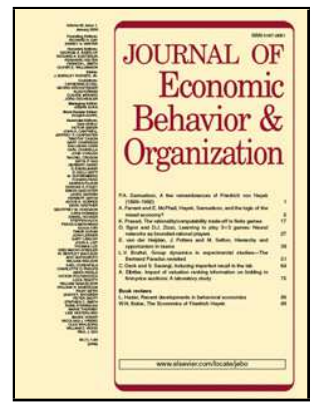

DOI: $\quad$ doi:10.1016/j.jebo.2011.01.006

Reference: $\quad$ JEBO 2666

To appear in: Journal of Economic Behavior \& Organization

Received date: $\quad 12-10-2010$

Revised date: $\quad$ 7-1-2011

Accepted date: $\quad 11-1-2011$

Please cite this article as: Binswanger, J., Dynamic decision making with feasibility goals: A procedural-rationality approach, Journal of Economic Behavior and Organization (2008), doi:10.1016/j.jebo.2011.01.006

This is a PDF file of an unedited manuscript that has been accepted for publication. As a service to our customers we are providing this early version of the manuscript. The manuscript will undergo copyediting, typesetting, and review of the resulting proof before it is published in its final form. Please note that during the production process errors may be discovered which could affect the content, and all legal disclaimers that apply to the journal pertain. 


\title{
Dynamic decision making with feasibility goals: A procedural-rationality approach*
}

\author{
Johannes Binswanger $^{\dagger}$
}

January 7, 2011

\begin{abstract}
This paper proposes a new framework of intertemporal choice: decision making by means of so-called feasibility goals. These refer to the feasibility of certain target levels of consumption associated with specific future benchmark scenarios. The feasibility goals framework takes into account bounded/procedural rationality without stepping outside the realm of an optimization framework. The paper has two aims. First, it provides a general characterization of the feasibility goals framework. Second, it provides a simple life cycle model with feasibility goals to illustrate the applicability of the concept. The life cycle model is promising for better understanding individual portfolio choice.
\end{abstract}

Key words: Feasibility goals, planning, bounded/procedural rationality, life cycle saving, portfolio choice.

JEL classification: D03, D81, D91.

*I am grateful to Lans Bovenberg, Simon Gächter, David Laibson, Wieland Müller, Manuel Oechslin, Ward Romp, Eytan Sheshinski, and seminar participants at the Austrian National Bank, at the University of Nottingham, the ENABLE Symposium on Economics and Psychology, Netspar, the 10th Annual Research Conference of the Dutch National Bank, and Tilburg University for helpful comments. All errors are my own.

${ }^{\dagger}$ Department of Economics, CentER and Netspar, Tilburg University, P.O. Box 90153, 5000 LE Tilburg, The Netherlands; j.binswanger@uvt.nl. 


\section{Introduction}

Most economic models rely on the assumption of unbounded rationality. This may be natural in the case of simple choices. The plausibility of this assumption has been questioned, however, in the case of complex dynamic decisions under uncertainty such as portfolio choice or life cycle saving (Pemberton, 1993; Thaler, 1994; Laibson et al., 1998). Advocates of the assumption of unbounded rationality point out that this assumption should not be taken literally. Rather, people are meant to behave as if they had unbounded cognitive capacities. They argue that people have been led to make rational optimizing decisions thanks to learning. This would happen in the same way as a billiard player learns to hit balls precisely without mastering the differential equations describing the balls' movements (Friedman, 1953). However, two recent papers (Lettau and Uhlig, 1999; Allen and Carroll, 2001) have put into question whether, from a theoretical perspective, boundedly rational agents can plausibly learn the type of dynamic behavior predicted by models of unbounded rationality. Barberis and Thaler (2003), Campbell (2006), and, in particular, Choi et al. (2009) provide empirical evidence that many people fail to learn unboundedly rational behavior in the domain of portfolio choice.

In the presence of uncertainty, a key feature of decision making under unbounded rationality is the requirement of a complete action plan. This plan specifies an optimal action at each future node of an event tree that may be reached with positive probability. It is noteworthy that, in the absence of such a plan, an expected utility function could not be evaluated and would thus be vacuous. Pre-specifying actions for all possible future circumstances is therefore an essential element of the standard model. Under realistic circumstances, the requirement of a complete action plan makes decision making according to the standard model very complex (Pemberton, 1993). Realistic circumstances entail the existence of transitory and permanent shocks to income, of liquidity constraints and the opportunity to invest in multiple financial assets.

If the complete plan of optimal actions - according to the standard model - had a simple structure, then it would be straightforward for agents to learn to behave "as if" 
following the standard model. However, Allen and Carroll (2001) have come up with an astonishing finding: under plausible assumptions, it would take an agent about one million years to learn a reasonable approximation to behavior as predicted by the standard model. This suggests that its predicted behavior is indeed complex under realistic circumstances.

This conclusion is further underlined by the observation that the standard model predicts a rather counter-intuitive (to those not familiar with the theory) pattern of portfolio choice over the life cycle. Typically, the young (at ages below 40) would invest 100 percent of their savings in stocks. Thereafter, the share of savings invested in stocks would gradually decrease to about 50 percent around retirement (Cocco et al., 2005; Gomes and Michaelides, 2005). In contrast, empirically, equity shares are very low for the young and then increase to a moderate level (see Section 5.2). The fact that the prediction of the standard model differs markedly from the empirical pattern gives rise to a puzzle.

In light of the above, a natural first step towards a model that describes decision making of boundedly rational agents is to eliminate the requirement of complete contingent planning. In order to deviate from the classical model in only one direction, it is valuable to continue relying on the other pillars of the standard model: preferences, constraints and optimization. In this paper, I put forward the framework of so-called feasibility goals that does exactly this.

In the feasibility goals framework, an individual has certain target consumption levels that she wants to remain feasible in certain future benchmark scenarios. For instance, an individual may choose her current consumption such that, in a future worst-case scenario, her minimum feasible future consumption is a certain proportion of her current consumption.

The essential property of feasibility goals is that meeting them does not pre-determine actual future decisions, unlike in the case of the standard model. To understand this, suppose that an agent has the feasibility goal of consuming at least 60 percent of current consumption in a worst-case scenario next period. What she will actually consume if the worst-case scenario materializes in that period is left open. This decision will be made 
only once this scenario has materialized. She may choose an actual consumption level that turns out to be higher or lower than 60 percent of the level of consumption in the previous period. However, having pursued the feasibility goal in the previous period, she ensures that she ends up with the possibility to consume at least 60 percent of her previous consumption in a worst-case scenario. In other words, the agent is prepared for a worst-case scenario.

The fact that ex-post choices may not be equal to ex ante feasibility goals for a particular scenario does not reflect dynamic inconsistency. The reason is that achieving the feasibility goal does not mean pre-specifying any future choice. Rather, it means preparing for bad future circumstances. The fact that decisions are made prudently, but sequentially "on the spot," reflects the principle of procedural rationality that characterizes actual planning.

This paper consists of two parts. In the first part, I introduce the concept of a feasibility goal. This is first done in an intuitive way by means of a few simple examples. I then provide a formal characterization of the feasibility goals framework. The second part of the paper presents a specific tractable life cycle model with feasibility goals that is calibrated and compared to the data. The aim of this second part is to show that the feasibility goals approach has bite for applied work. The analysis of the life cycle model shows that forward looking behavior when guided by simple feasibility goals is sufficient for very smooth consumption profiles. Furthermore, the life cycle model explains empirical asset allocation patterns better than a large variety of existing models.

The paper is organized as follows. Section 2 outlines the related literature. Section 3 introduces the concept of feasibility goals in an intuitive way. Section 4 characterizes the concept formally. Section 5 presents a life cycle model with feasibility goals. Section 6 concludes. 


\section{Related Literature}

This paper contributes to the small but growing literature of intertemporal models in behavioral economics. Existing behavioral life cycle models include the mental accounting model of Shefrin and Thaler (1988), models of hyperbolic discounting (Laibson, 1997), and the loss aversion model of Bowman et al. (1999). The aim of this literature is to study the effect of psychological biases on savings behavior. In this paper, the issue is bounded rationality rather than psychological biases.

Gabaix and Laibson $(2000,2005)$ provide models of bounded rationality where agents use short-cuts when working through a decision tree. In particular, agents remove branches with low probability. An important difference between the feasibility goals framework and the models of Gabaix and Laibson is that they study decisions where a choice is made only once at the beginning of a probabilistic event three. In contrast, the feasibility goals framework applies to dynamic situations with a multitude of follow-up decisions. Bolton and Faure-Grimaud (2009) provide a model of procedural rationality in dynamic decision making to analyze endogenous information processing. In contrast, the feasibility goals framework describes prudent and forward-looking decision making taking limited information processing capabilities as exogenously given.

Pemberton (1993) develops a life cycle model that shares the spirit of feasibility goals models. However, neither does he provide a general characterization of the feasibility goals framework nor does he analyze asset allocation.

\section{An Intuitive Introduction to Feasibility Goals}

I begin the analysis by developing the concept of feasibility goals in an intuitive way. This prepares the formal characterization of the concept in Section 4. A feasibility goal intrinsically relates to the notion of a (future) scenario. Imagine that a scenario refers simply to one specific path through an event tree. It starts at current time $t$. Denote the (deterministic) terminal time by $T$ and denote a particular scenario starting at $t$ by $s_{t}$. 
A scenario $s_{t}$ "passes" $T-t$ time periods and nodes of the event tree and ends at time T. Naturally, there is uncertainty about the future if and only if there is more than one scenario from a node at $t$ onwards.

A feasibility goal assigns to each point in time along the scenario $s_{t}$ a level of consumption that is to be affordable. A feasibility goal does not specify any actual future action. Thus, it does not constitute a contingent plan. It only specifies feasibility of a certain level of consumption along $s_{t}$.

How the feasibility goal concept works is best understood by means of a set of simple examples. The below examples are intentionally kept stylized in order to illustrate the concept. In Section 5 below, I present a more sophisticated model of life cycle saving and asset allocation that illustrates the usefulness of the concept for applied work and of its empirical bite.

\subsection{Example 1: An Absolute Feasibility Goal for a Worst-Case Scenario}

Consider an agent living for three periods. Assume that the agent has an endowment of $y_{1}$ in the first period. Furthermore, she can transfer resources to the future by saving. The gross interest rate is equal to $r$.

This first example of a feasibility goal is a particularly simple one. It entails affordability of specific absolute consumption levels in a future worst-case scenario. These are $\bar{c}_{2}$ for the second and and $\bar{c}_{3}$ for the third period, respectively. The worst-case scenario is defined as the path through the event tree where future income is lowest. Consider the extreme case that the worst-case scenario entails no income in the future at all (except from current savings). Assume that $y_{1}>\frac{\bar{c}_{2}}{r}+\frac{\bar{c}_{3}}{r^{2}}$, i.e. the present value of minimum lifetime income exceeds the present value of the future consumption levels associated with the feasibility goal. Assume finally that the agent has a preference ordering according to which she likes most the choice with the highest level of current consumption, out of all 
choices that allow for meeting the feasibility goal. ${ }^{1}$

In the first period, the agent's decision problem looks as follows. Denote first-period consumption by $c_{1}$. The agent meets her feasibility goal in the first period if and only if $y_{1}-c_{1} \geq \frac{\bar{c}_{2}}{r}+\frac{\bar{c}_{3}}{r^{2}}$, i.e. $c_{1} \leq y_{1}-\frac{\bar{c}_{2}}{r}-\frac{\bar{c}_{3}}{r^{2}}$. This means that savings in the first period are sufficient for financing the feasibility goal. Whenever $c_{1}>y_{1}-\frac{\bar{c}_{2}}{r}-\frac{\bar{c}_{3}}{r^{2}}$, then it is not feasible to consume at least $\overline{c_{2}}$ in the second and $\overline{c_{3}}$ in the third period and so the feasibility goal is not achieved. Since the decision maker maximizes current consumption subject to meeting the feasibility goal, she chooses $c_{1}=y_{1}-\frac{\bar{c}_{2}}{r}-\frac{\bar{c}_{3}}{r^{2}}$ and saves the rest of her income.

Meeting the feasibility goal does not pre-specify any decisions in the second (or third) period. Instead, the agent follows a procedural rationality approach. She simply applies the same decision mechanism again upon entering period two or three. Since she made a prudent forward-looking decision in the first period, it is assured that her later consumption will indeed not fall below a certain lower bound.

Specifically, the agent enters the second period with a level of resources of $X_{2}$. The latter depends on the particular scenario that has emerged. In the worst-case scenario, it consists only of savings. Otherwise, it may also comprise other sources of income. Whatever $X_{2}$ amounts to, the agent's feasibility goal calculus, as applied in the second period, requires to consume at least $\bar{c}_{3}$ in the third period. Since, in the worst-case scenario, the agent earns no income apart from returns on her savings in the third period, she needs to save at least $\frac{\bar{c}_{3}}{r}$ in order to meet the feasibility goal. Since she maximizes current consumption subject to meeting the feasibility goal, she will choose a secondperiod consumption of $c_{2}=X_{2}-\frac{\bar{c}_{3}}{r}$. In the third period the agent just consumes whatever resources are left.

As has been illustrated by this example, intertemporal decision making by means of feasibility goals means applying the same decision algorithm every period anew. Apart from this, there is no functional link between decision making at different points in time,

\footnotetext{
${ }^{1}$ Of course, other preference orderings would be possible.
} 
unlike in the case of a contingent action plan in the standard model. However, by their very nature, feasibility goals allow for making prudent and forward-looking decisions in spite of this seemingly missing link. To see this, note that the agent will indeed have the possibility of consuming at least $\bar{c}_{2}$ in the second period and $\bar{c}_{3}$ in the third.

Decision making by means of feasibility goals does not lead to dynamic inconsistency. The reason is that achieving feasibility goals does only mean to ensure feasibility of certain future actions, not pre-specifying any actual future consumption levels. Thus, there is, by the very nature of the feasibility goals concept, no room for dynamic inconsistency. Rather, it reflects a procedural-rationality approach.

Example 1 is particularly simple and stylized, but it provides a clean illustration of how the feasibility goals concept works.

\subsection{Example 2: A Relative Feasibility Goal for a Worst-Case Scenario}

In this example everything is identical to the first one except that the feasibility goal is now relative in nature. This relative feasibility goal entails consuming at least a fraction $\alpha$ of current consumption in every future period if a worst-case scenario emerges. It is natural to assume that $0<\alpha \leq 1$, although this restriction is not necessary from a technical point of view.

In the first period, the feasibility goal is again met if and only if savings are sufficiently high, i.e. $y_{1}-c_{1} \geq \frac{\alpha c_{1}}{r}+\frac{\alpha c_{1}}{r^{2}}$, or $c_{1} \leq\left(1+\frac{\alpha}{r}+\frac{\alpha}{r^{2}}\right)^{-1} y_{1}$. If the decision maker's aim is to maximize current consumption subject to meeting the feasibility goal then she will choose $c_{1}=\left(1+\frac{\alpha}{r}+\frac{\alpha}{r^{2}}\right)^{-1} y_{1}$ and save the rest of her income.

In the second period, the agent ends up with a level of resources $X_{2}$. She meets her feasibility goal in the second period if $X_{2}-c_{2} \geq \frac{\alpha c_{2}}{r}$. If she maximizes current consumption subject to achieving the feasibility goal, she chooses $c_{2}=\frac{r}{\alpha+r} X_{2}$. In the third period, the agent will consume whatever resources are left. 


\subsection{Example 3: Feasibility Goals for a Worst-Case and a Normal Scenario}

This example illustrates that it is straightforward to have multiple feasibility goals. The first feasibility goal coincides with the one of the previous example and may be dubbed safety goal. The second feasibility goal relates to a "normal" or "average" scenario. Specifically, the agent's goal is the feasibility of a sequence of consumption levels that increases by at least a factor $\beta \geq 1$ from period to period. In other words, this means feasibility of a higher standard of living in the future in a normal scenario. There are again three periods. Non-financial income in the normal scenario is $y^{n}$ in both future periods. In the worst-case scenario non-financial income is zero as in the previous two examples.

In the first period, meeting the normal-scenario goal means assuring feasibility of a consumption profile of the form $\left(c_{1}, \beta c_{1}, \beta^{2} c_{1}\right)$. This requires that $c_{1}+\frac{\beta c_{1}}{r}+\frac{\beta^{2} c_{1}}{r^{2}} \leq$ $y_{1}+\frac{y^{n}}{r}+\frac{y^{n}}{r^{2}} \equiv Y^{n}$ or $c_{1} \leq\left(1+\frac{\beta}{r}+\left(\frac{\beta}{r}\right)^{2}\right)^{-1} Y^{n}$. In contrast, the feasibility goal associated with the worst-case scenario requires that $c_{1} \leq\left(1+\frac{\alpha}{r}+\frac{\alpha}{r^{2}}\right)^{-1} y_{1}$ (see Example 2 above). If the agent chooses the highest level of first-period consumption that is compatible with both goals, she chooses $c_{1}=\min \left\{\left(1+\frac{\beta}{r}+\left(\frac{\beta}{r}\right)^{2}\right)^{-1} Y^{n},\left(1+\frac{\alpha}{r}+\frac{\alpha}{r^{2}}\right)^{-1} y_{1}\right\}$.

In the second period, achieving the normal-scenario goal requires that $c_{2}+\frac{\beta c_{2}}{r} \leq$ $X_{2}+\frac{y^{n}}{r}$. Achieving the feasibility goal for the worst-case scenario requires $c_{2}+\frac{\alpha c_{2}}{r} \leq X_{2}$. The highest level of second-period consumption that allows the agent to meet both goals is given by $c_{2}=\min \left\{\frac{r}{\beta+r}\left(X_{2}+\frac{y^{n}}{r}\right), \frac{r}{\alpha+r} X_{2}\right\}$ 


\section{A Formal Characterization of the Feasibility Goals Framework}

\subsection{Feasibility Goals}

Let us start with a formal description of an event tree. Time $t$ runs from one to $T$. At $t=1$, an event $e_{1} \in \mathcal{E}_{1}$ occurs, where $\mathcal{E}_{1}$ is the set of all possible events at $t=1$. Given the realization of a particular $e_{1} \in \mathcal{E}_{1}$, the set of possible follow-up events for $t=2$ is generally a function of the realization of $e_{1}$. It is denoted $\mathcal{E}_{2}\left(e_{1}\right)$ and has a typical element $e_{2}$. Iterating further, a particular node of the event tree at time $t$ is characterized by the event $e_{t} \in \mathcal{E}_{t}\left(e_{t-1}\right)$, where $e_{t-1} \in \mathcal{E}_{t-1}\left(e_{t-2}\right), e_{t-2} \in \mathcal{E}_{t-2}\left(e_{t-3}\right), \ldots, e_{2} \in \mathcal{E}_{2}\left(e_{1}\right), e_{1} \in \mathcal{E}_{1}$. For a more compact notation, it is convenient to define a history $h_{t}:=\left(e_{1}, e_{2}, \ldots, e_{t}\right)$, $e_{1} \in \mathcal{E}_{1}, \ldots, e_{2} \in \mathcal{E}_{2}\left(e_{1}\right), \ldots, e_{t} \in \mathcal{E}_{t}\left(e_{t-1}\right)$ and to denote the set of possible events at $t$ by $\mathcal{E}_{t}\left(h_{t-1}\right)$.

Given a history $h_{t}$, a scenario at time $t$ is defined as a sequence of follow-up events of the history $h_{t}$. Generically, a scenario is thus described as a sequence $\left(e_{t+1}, e_{t+2}, \ldots, e_{T}\right)$ with $e_{t+1} \in \mathcal{E}_{t+1}\left(h_{t}\right), e_{t+2} \in \mathcal{E}_{t+2}\left(e_{t+1}\right), \ldots, e_{T} \in \mathcal{E}_{T}\left(e_{T-1}\right)$. The set of all possible scenarios following history $h_{t}$ is denoted by $\mathcal{S}_{t}\left(h_{t}\right)$. The typical element of this set is denoted by $s_{t}$.

Before defining feasibility goals, we need to formally describe sets of feasible actions, i.e. dynamic "budget constraints." ${ }^{2}$ Denote $\mathcal{A}_{t}\left(h_{t}\right) \subset \mathcal{R}^{n}$ the set of potentially feasible actions at time $t$, given history $h_{t}$. The typical element of $\mathcal{A}_{t}\left(h_{t}\right)$ is $a_{t}$ and may be a multi-dimensional vector. Denote by $\mathcal{B}\left(h_{t}\right) \subset \mathcal{A}_{t}\left(h_{t}\right)$ the set of all feasible actions, i.e. a "budget" set. The budget set in period $t+1$ generally depends on the action chosen at $t$, as well as on the event that has materialized in $t+1$. Generically, one would describe this budget set as $\mathcal{B}_{t+1}\left(h_{t}, e_{t+1}, a_{t}\right)$, where $e_{t+1} \in \mathcal{E}_{t+1}\left(h_{t}\right), a_{t} \in \mathcal{B}_{t}\left(h_{t}\right)$. Similarly, the budget set in $t+2$ would be described as $\mathcal{B}_{t+2}\left(h_{t}, e_{t+2}, a_{t+1}\right)$, where $e_{t+2} \in \mathcal{E}_{t+2}\left(e_{t+1}\right)$,

\footnotetext{
${ }^{2}$ These may be proper budget constraints or represent any other restrictions on an agent's actions that may be relevant.
} 
$e_{t+1} \in \mathcal{E}_{t+1}\left(h_{t}\right), a_{t+1} \in \mathcal{B}_{t+1}\left(h_{t}, e_{t+1}, a_{t}\right), a_{t} \in \mathcal{B}_{t}\left(h_{t}\right)$. In order to simplify notation, I will use a shortcut and describe the budget set $\tau$ periods ahead as $\mathcal{B}_{t+\tau}\left(s_{t}, a_{t+\tau-1}\right)$, where $a_{t+\tau-1} \in \mathcal{B}_{t+\tau-1}\left(s_{t}, a_{t+\tau-2}\right), a_{t+\tau-2} \in \mathcal{B}_{t+\tau-2}\left(s_{t}, a_{t+\tau-3}\right)$ etc. Similarly, $\mathcal{A}_{t+\tau}\left(s_{t}, a_{t+\tau-1}\right)$ is a shortcut for the set of potentially feasible actions at time $t+\tau$, given history $h_{t}$ and scenario $s_{t}$.

A feasibility goal is then defined as follows.

Definition 1 (Feasibility Goal) A feasibility goal $\hat{f}_{t}\left(s_{t}\right)$ for scenario $s_{t}$ is a mapping $\mathcal{S}_{t}\left(h_{t}\right) \rightarrow \mathcal{A}_{t+1}\left(s_{t}\right) \times \mathcal{A}_{t+2}\left(s_{t}\right) \times, \ldots, \times \mathcal{A}_{T}\left(s_{t}\right)$ assigning to $s_{t}$ a sequence of future target actions $\left(\hat{a}_{t+1}, \hat{a}_{t+2}, \ldots, \hat{a}_{T}\right)$. A feasibility goal is met at time $t$ if $a_{t}$ is such that $\hat{a}_{t+1} \in$ $\mathcal{B}_{t+1}\left(s_{t}, a_{t}\right), \hat{a}_{t+2} \in \mathcal{B}_{t+2}\left(s_{t}, \hat{a}_{t+1}\right), \ldots, \hat{a}_{T} \in \mathcal{B}_{T}\left(s_{t}, \hat{a}_{T-1}\right)$.

The "hats" on the actions $\hat{a}_{t+\tau}$ constituting the feasibility goal indicate that these may be different from the actions $a_{t+\tau}$ that are actually chosen at times $t+\tau$. It is important to note that, according to Definition 1, "meeting a feasibility goal" is a qualification of a current action $a_{t}$.

Let us illustrate this definition by means of Example 2 of the previous subsection. Consider the decision problem in period one. The action $a_{t}$ corresponds to the choice of consumption $c_{1}$. The feasibility goal for the worst-case scenario is $\left(\hat{a}_{t+1}, \hat{a}_{t+2}\right)=\left(\alpha c_{1}, \alpha c_{1}\right)$. For concreteness, suppose that $y_{1}=100, r=1, \alpha=.5$. Let us ask whether $c_{1}=50$ fulfills the feasibility goal. In case that the worst-case scenario emerges, this results in a level of resources of $(100-50) \times 1=50$ for the second period. (Recall that there is no nonfinancial income in the worst-case scenario.) The target consumption level for the second period for this scenario is $.5 \times 50=25$. A consumption level of 25 is thus feasible. It leaves another 25 of savings, leading to a level of resources of 25 in the third period in case of the worst-case scenario. This is just the target level specified by the feasibility goal. Thus, the choice $c_{1}=50$ does fulfill the feasibility goal in the first period. If we had $c_{1}=60$, then the level of resources in the second period would be 40 and the target level of consumption for the second period would be 30 . Thus, the target could still be met in the second period. However, there would remain savings of only 10 in the second period. 
This would lead to a level of resources of only 10 in the third period, not allowing for a target consumption of 30 . Thus, the choice $c_{1}=60$ would not fulfill the feasibility goal.

The case of Example 2 highlights the fact that, for the feasibility goal $\hat{f}_{t}\left(s_{t}\right)=$ $\left(\hat{a}_{t+1}, \hat{a}_{t+2}, \ldots, \hat{a}_{T}\right)$, the target actions $\hat{a}_{t+\tau}, 1 \leq \tau \leq T-t$ may be a function of the current actions $a_{t}$. In fact, whenever feasibility goals are relative goals with respect to current consumption, then the target actions $\hat{a}_{t+\tau}$ are functions of current action $a_{t}$. On the other hand, feasibility goals need not be functions of current actions. This is illustrated by Example 1.

\subsection{Preferences over Feasibility Goals}

Feasibility goals by themselves do not yet imply a specific choice. Rather, they partition feasible choices in period $t$ in a subset of choices that meet the feasibility goals and a subset that does not. A preference relation is required to rank the choices that do fulfill the feasibility goals.

In the feasibility goals framework, the domain of preferences is what I dub the feasibilitygoals induced choice (FIC) set. To state the formal definition of the FIC set, assume that there are $K$ feasibility goals as defined by Definition 1 related to $K$ scenarios. We have then:

Definition 2 (FIC set) Define $\mathcal{F}_{t} \subset \mathcal{B}_{t}$ to be the set of all current choices a that fulfill the $K$ feasibility goals. Using a shortcut notation, write the budget sets as $\mathcal{B}_{t+\tau}\left(s_{t}^{k}, a_{t}\right)$, $1 \leq \tau \leq T-t, 1 \leq k \leq K$. The feasibility-goals induced (FIC) choice $\mathcal{C}_{t}$ set is defined as the set

$$
\begin{aligned}
\mathcal{F}_{t} & \times\left[\mathcal{B}_{t+1}\left(s_{t}^{1}, a_{t}\right) \times \mathcal{B}_{t+2}\left(s_{t}^{1}, a_{t}\right) \times \ldots \times \mathcal{B}_{T}\left(s_{t}^{1}, a_{t}\right)\right] \\
\times & {\left[\mathcal{B}_{t+1}\left(s_{t}^{2}, a_{t}\right) \times \mathcal{B}_{t+2}\left(s_{t}^{2}, a_{t}\right) \times \ldots \times \mathcal{B}_{T}\left(s_{t}^{2}, a_{t}\right)\right] } \\
\times & \ldots \\
\times & {\left[\mathcal{B}_{t+1}\left(s_{t}^{K}, a_{t}\right) \times \mathcal{B}_{t+2}\left(s_{t}^{K}, a_{t}\right) \times \ldots \times \mathcal{B}_{T}\left(s_{t}^{K}, a_{t}\right)\right] }
\end{aligned}
$$

for which $a_{t} \in \mathcal{F}_{t}$.

Thus, the FIC set is the Cartesian product of the set of current actions that fulfill the 
relevant feasibility goals and the set of induced target actions. Recall that, in general, current actions may affect the target actions $\hat{a}_{t}+\tau \in \mathcal{B}_{t+\tau}$, constituting a feasibility goal. A simple instance of this is given by Examples 2 and 3 above. The elements of the FIC set $\mathcal{C}_{t}$ have the typical form

$$
\left(a_{t}, \hat{f}^{1}\left(a_{t} ; s_{t}^{1}\right), \hat{f}^{2}\left(a_{t} ; s_{t}^{2}\right), \ldots, \hat{f}^{K}\left(a_{t} ; s_{t}^{K}\right)\right)
$$

The next definition characterizes preferences in the feasibility goals framework.

Definition 3 In the feasibility goals framework, preferences $\succeq_{t}^{F G}$ are a complete and transitive relation over the $F I C$ set $\mathcal{C}_{t}$.

In contrast to the standard microeconomic framework, preferences are only relevant for ranking choices within the narrow FIC set, not the overall choice set. In other words, the agents has a preference order only over those choices that fulfill the feasibility goals. This reflects bounded rationality.

It is noteworthy that preferences may not simply be defined over $\mathcal{F}_{t}$ but over the larger set $\mathcal{C}_{t}{ }^{3}$ This simply means that preferences $\succeq_{t}^{F G}$ may not simply be preferences over current actions that fulfill the feasibility goals. Rather, preferences may also directly be responsive to the induced level of future target actions $\hat{a}_{t+\tau}$. The life-cycle model in Section 5 will provide an instance of this.

As in the case of standard microeconomic theory, it is required that the preference relation $\succeq_{t}^{F G}$ is complete and transitive over the FIC set $\mathcal{C}_{t}$. However, this rationality requirement for preferences over $\mathcal{C}_{t}$ is weaker than in the case of the standard model with full contingent planning. In the standard model, the domain of preferences is the Cartesian product of all current and contingent future actions in all possible states of the world. In contrast, in the feasibility goals framework, the domain of the preference relation $\succeq_{t}^{F G}$ is only the restricted set $\mathcal{C}_{t}$. It is restricted by the fact that an agent only

\footnotetext{
${ }^{3}$ Of course, the framework allows for the possibility that the domain of preferences is, in fact, only $\mathcal{F}_{t}$; this simply provides a special case. See below.
} 
considers choices that meet the feasibility goals in the first place. Second, concerning the future, preferences are only about feasibility goals, not actually planned contingent actions. Both restrictions reflect procedural rationality.

To illustrate the domain $\mathcal{C}_{t}$ of preferences, consider again Example 3 from the previous subsection. It has been assumed that the agent prefers the choice with the highest level of current consumption among all choices fulfilling the feasibility goals. There, we have, for the first period,

$$
\mathcal{F}_{1}=\left\{c_{1}: c_{1} \leq \min \left[\left(1+\frac{\beta}{r}+\left(\frac{\beta}{r}\right)^{2}\right)^{-1} Y^{n},\left(1+\frac{\alpha}{r}+\frac{\alpha}{r^{2}}\right)^{-1} y_{1}\right]\right\}
$$

Furthermore, the elements of the set $\mathcal{C}_{1}$ have the form

$$
\left(c_{1},\left(\alpha c_{1}, \alpha c_{1}\right),\left(\beta c_{1}, \beta^{2} c_{1}\right)\right)
$$

where $c_{1} \in \mathcal{F}_{1}$. Denote the elements of $\mathcal{C}_{1}$ by $\mathbf{c}_{1}$. Consider $\mathbf{c}_{1}^{\prime}, \mathbf{c}_{1}^{\prime \prime} \in \mathcal{C}_{1}$ with first elements $c_{1}^{\prime}$ and $c_{1}^{\prime \prime}$, respectively. In the case of Example 3, we have

$$
\mathbf{c}_{1}^{\prime} \succeq_{1}^{F G} \mathbf{c}_{1}^{\prime \prime} \text { iff } c_{1}^{\prime} \geq c_{1}^{\prime \prime}
$$

In this example, $\succeq_{1}^{F G}$ is, in fact, a preference relation only over $\mathcal{F}_{1}$.

It is noteworthy that the fact that the feasibility goals framework continues to rely on the preference concept, although in a very restricted sense, distinguishes it from a rule-of-thumb approach. The latter would lead to a complete departure from classical decision theory, whereas the feasibility goals framework deviates insofar as there is no contingent action plan. The contingent action plan is substituted by a procedural rationality approach. This notwithstanding, the feasibility goals framework still relies on the classical pillars: preferences, constraints and optimization. 


\section{A Life Cycle Model with Feasibility Goals}

\subsection{The Model}

In this section I present a model of life cycle saving and asset allocation. The main purpose of this section is to illustrate the applicability of the feasibility goals framework and its empirical bite. More specifically, the life cycle model demonstrates that, in spite of only procedural rationality, the resulting consumption path is very smooth. Second, the model shows that the feasibility approach is promising for better understanding individual portfolio choice. As will be discussed below, existing models have major difficulties explaining how portfolio structures vary over the life cycle. ${ }^{4}$

Consider an agent who, at each time $t$, makes a decision about how much to consume, how much to invest in a safe asset, called bond, and and in a risky asset, called stock. Time $t$ runs from 1 to $T$. Consumption is denoted by $c_{t}$, bond holdings by $b_{t}$ and stock holdings by $s_{t}$. In each period, budget constraints are given by the two equations

$$
\begin{aligned}
& c_{t}+b_{t}+s_{t}=X_{t}, \\
& X_{t}=y_{t}+b_{t-1} r+s_{t-1} \rho_{t} .
\end{aligned}
$$

$X_{t}$ denotes total available resources in $t$ (where $X_{1}$ is given). The variable $y_{t}$ denotes labor income, $r$ denotes the risk-free interest rate and $\rho_{t}$ stock returns in period $t$. Both $y_{t}$ and $\rho_{t}$ are random variables. The distribution of $y_{t}$ may depend on previous realizations as well as on $t$ itself. Thus, in expectations, $y_{t}$ may follow a typical hump-shaped profile over the life cycle (see Appendix). For simplicity, I assume that $\rho_{t}$ is iid.

Following much of the literature (see e.g. Cocco et al., 2005), I assume that bond and stock holdings must be nonnegative, i.e. $b_{t} \geq 0, s_{t} \geq 0$.

There are two feasibility goals relating to two different scenarios: a worst-case and a

\footnotetext{
${ }^{4}$ The model described below is discussed at greater length in a companion working paper (Binswanger, 2009) and for some specific details the reader is referred to the latter. The below discussion benefits from the concepts developed in Section 4 and the discussion here is more streamlined than in Binswanger (2009).
} 
normal scenario (cf. Example 3 above). The goal referring to the worst-case is dubbed safety goal. For any period $t$, the safety goal is given by

$$
\left(\alpha_{1} c_{t}, \alpha_{2} c_{t}, \ldots, \alpha_{T-t} c_{t}\right)
$$

Thus, it entails feasibility of (potentially differing) target fractions $\alpha_{\tau}$ of current consumption in the future in a worst-case scenario. This is a simple extension of the feasibility goal in Examples 2 and 3 above.

The second goal relates to a normal scenario and is reminiscent of the normal-scenario goal in Example 3. It is modeled in a more flexible way, however. This goal is dubbed the future standard of living (FSL) goal. It entails a standard of living of $c^{+}$from a future period $T^{*}$ on for the case that the normal scenario materializes. Specifically, the FSL goal $c^{+}$is defined as a level of consumption that is feasible from $T^{*}$ onward if (i) consumption is equal to the current level $c_{t}$ between $t$ and $T^{*}$, (ii) the normal scenario materializes, and (iii) savings are invested in a portfolio of bonds and stocks between $t$ and $T^{*}$ in such a way that the safety goal is always met given a level of consumption of $c_{t}$.

Formally, the FSL goal is stated as

$$
\left(c_{t}, c_{t}, \ldots, c^{+}, c^{+}, \ldots\right)
$$

where $c_{t}$ appears $T^{*}-t-1$ times and $c^{+}$appears $T-T^{*}+1$ times. The FSL goal $c^{+}$is determined as the constant consumption stream that can be financed by $X_{T^{*}}^{n}$, where the superscript indicates the normal scenario. $X_{T^{*}}^{n}$ is determined by the accumulation process

$$
X_{t+\tau+1}^{n}=b_{t+\tau} r+s_{t+\tau} \rho^{n}+y_{t+\tau+1}^{n},
$$

where $\rho^{n}$ denotes stock returns in the normal scenario and $0 \leq \tau \leq T^{*}-t-1$. Furthermore,

$$
b_{t+\tau}+s_{t+\tau}=X_{t+\tau}^{n}-c_{t}
$$


and the choice $\left(c_{t}, b_{t+\tau}, s_{t+\tau}\right)$ is such that it fulfills the safety goal for all $\tau, 0 \leq \tau \leq$ $T^{*}-t-1$, along the projected normal scenario.

The relevant preference order $\succeq_{t}^{F G}$ is defined as follows.

Definition 4 (Life Cycle Preferences) Among two choices $z=\left(c_{t}, b_{t}, s_{t}\right), z^{\prime}=\left(c_{t}^{\prime}, b_{t}^{\prime}, s_{t}^{\prime}\right)$ that fulfill all relevant safety goals, $z$ is preferred to $z^{\prime}$ if $z$ leads to a higher value of the quasi-utility function $u^{F G}=c_{t}\left(c^{+}\right)^{\gamma}, \gamma>0$.

In contrast to the normal-scenario goal in Example 3, there is thus an explicit trade-off between current consumption and the FSL goal $c^{+}$.

There are three ways in which bounded rationality shapes preferences $\succeq_{t}^{F G}$. First, the agent only considers two future scenarios. Second, the agent immediately eliminates all choices that do not allow for meeting the safety goal. Third and most crucially, all arguments of the preference relation that refer to the future are feasibility goals (i.e. the safety goals $\alpha_{\tau} c_{t}$ and $c^{+}$). There is no direct functional relationship between these goals and actual future decisions. This reflects that there is no contingent planning. However, meeting the feasibility goals means that a certain minimum level of consumption in the worst-case scenario and a certain (higher) standard of living in the normal scenario are indeed feasible in the future. Thus, the agent is prepared for the future thanks to her feasibility goals.

\subsection{Calibrating the Model}

I now proceed by calibrating the model. This allows for getting an insight about the smoothness of the resulting consumption profile and whether the model has any power to explain the empirical asset allocation profile.

I consider a setup where an agent starts working at age 21, retires at the beginning of age 66 , and dies with certainty after age $85 .{ }^{5}$ For the safety goal, I use the one-parameter

\footnotetext{
${ }^{5}$ Considering uncertain lifespans is beyond scope of this paper.
} 
specification $\alpha_{\tau}=\left(\alpha^{*}\right)^{\tau}$ where $\alpha^{*}$ is set to $.97 .{ }^{6}$ This specification has the advantage of being very parsimonious. Furthermore, it captures the idea that decision makers may be less concerned, or may think less carefully, about negative events in the far-distant future than in the near future. I set $\gamma$ to .85 . Finally, I set $T^{*}=(t+T) / 2$, i.e. it refers to the middle of the remaining life time.

Non-financial income is calibrated using estimations of the labor income profile for high-school graduates reported in Cocco et al. (2005). Labor income is subject to permanent and transitory shocks. These are assumed to be log-normally distributed. There is also a medical expenditure shock $M$ that reduces non-financial income $y_{t}$ during retirement to $M y_{t}$. It is realized upon retirement and is fully permanent. The expected value of $M$ is one and its lower bound $\underline{M}$ is set to .5. Details about the calibration of non-financial income can be found in the Appendix. Real bond returns are assumed to be risk-free and to equal 2 percent per year. Stock returns are assumed to be iid over time and lognormally distributed with an expected annual return of 6 percent and a standard deviation of .157.

The normal scenario is defined as the one corresponding to a state of the world where labor income, stock returns and the medical shock take on their expected values at all future dates, given current information. The worst-case scenario is defined as the one corresponding to future states of the world where labor income shocks and stock returns take on the value of their first percentile. Furthermore, the medical shock $M$ takes on the value $\underline{M}$ upon retirement. The calibrations are run for the case where, ex post, the normal scenario materializes.

Figures 1 and 2 present the simulation results. The solid line in Figure 1 refers to consumption. The dashed line represents non-financial income. The dotted line represents total income including financial income, which is endogenous since it depends on the agent's savings and asset allocation choices. Figure 2 shows calibrated equity shares. Equity shares are defined as the ratio of total stock holdings to total financial assets, i.e.

\footnotetext{
${ }^{6}$ Calibrations for parameter values others than the ones discussed here can be found in Binswanger (2009). The results discussed below do not hinge upon the specific parameter values chosen.
} 
$s_{t} /\left(b_{t}+s_{t}\right)$.

Figure 1 shows that consumption follows income very closely until the age of about 35. Furthermore, the level of savings is very low during these years and there is only a small "buffer stock." Thereafter, savings start to increase and consumption levels off. The dominant savings motive is preparation for retirement. ${ }^{7}$

The first important observation is that the consumption profile is virtually as smooth as it can be in the presence of binding liquidity constraints. In fact, the predicted profile resembles very closely the shape of consumption profiles for expected utility models with buffer stock saving (Carroll, 1997; Cocco et al., 2005). Thus, the analysis reveals that no complex contingent action plan is necessary in order to achieve consumption smoothing. A key insight is thus that the simple procedural-rationality approach of the feasibility goals model is sufficient for consumption smoothing.

The second key observation is that the feasibility goals model matches the shape of empirical equity shares better than existing models. Table 1 shows how empirical equity shares vary over age, based on the 2004-wave of the Survey of Consumer Finances (SCF). These equity shares contain direct as well as indirect equity holdings through mutual funds and retirement accounts. Empirical equity shares are very low under the age of 30 . Thereafter they increase until the age of 55 and decrease slightly thereafter. ${ }^{8}$

Existing models have major difficulties of explaining this empirical pattern. Equity shares are typically predicted to be close to 100 percent until the age of about 40 . Thereafter, they decrease until retirement, where they reach a level of about 50 percent, on average (Cocco et al., 2005). The prediction that equity shares are far higher than empirical estimates over a large part of the life cycle is very robust across existing models. Very high equity shares arise even when taking into account the possibility of very low re-

${ }^{7}$ After the age of 70 , consumption increase further whereas equity shares continue to decrease. The model is less suited, however, to predict savings behavior during later phases of retirement since important determinants of choices during that phase are missing. These include savings for nursery homes and bequest motives.

${ }^{8}$ It is noteworthy that Table 1 is based on observations from only one particular year. Therefore, the shape of the equity share profile cannot be biased by what is known as time effects. 
alizations of labor income or endogenous borrowing constraints (Cocco et al., 2005), fixed costs of stock market participation and Epstein-Zin preferences (Gomes and Michaelides, 2005) or habit formation (Gomes and Michaelides, 2003, Polkovnichenko, 2007).

The feasibility goals model captures well the general shape of the empirical equity shares profile. In particular, equity shares are predicted to be very low until the thirties. Thereafter, they increase to a level that is somewhat higher than observed in the data. At the age of 55, equity shares start to decrease slightly. In sum, the feasibility goals model explains the data better than a large variety of existing models.

The intuition behind the predicted equity share profile is as follows. The young face a substantial amount of career uncertainty. The downside risk associated with future income declines substantially over time. This happens because the effects of a sequence of low realizations of the permanent income shock accumulate over time (see equation (11) in the Appendix). Hence, the uncertainty about future income and its downside risk is the lower, the less periods there are between a current period $t$ and retirement. Put simply, more things can still go wrong for a 25-year old than for a 50-year old.

After the age of 45 , the medical expenditure shock $M$ comes into play. To understand this, recall that agents do not want to let their future feasible standards of living fall below $\left(\alpha^{*}\right)^{i} c_{t}$, where $i$ represents the distance between the current and a particular future period. In early ages, retirement and hence the date of the realization of the medical expenditure shock lie far ahead in the future, such that $\alpha^{R-t}$ is very low, where $R$ denotes retirement age. When agents get closer to retirement, $\alpha^{R-t}$ increases. This prevents equity shares from further increasing and eventually leads to a decrease in stock holdings and an increase in bond holdings. This leads to a decrease of the equity share.

\section{Conclusion}

This paper puts forward a new approach of modeling intertemporal choice: decision making by means of feasibility goals. This approach represents a natural alternative to the standard modeling approach for two reasons. First, it takes into account bounded or 
procedural rationality by eliminating the requirement of a full contingent action plan. Second, the framework stays close to the standard approach. It is still based on an optimization framework relying on the concepts of preferences and constraints, unlike, for instance, a rule-of-thumb approach.

This paper has characterized the conceptual properties of feasibility goals models. Furthermore, it provides a comparatively simple life cycle model that is illustrates the applicability of the concept. The key insights from the life cycle analysis are that: (i) the resulting consumption profiles are virtually as smooth as for the standard model; (ii) empirical patterns of asset allocation are far better explained than by the standard model. 


\section{Appendix: Calibration of Income Process}

Following Cocco et al. (2005), I assume that

$$
y_{t}=F_{t} P_{t} V_{t}
$$

for $1 \leq t<R$, where $R$ denotes the age of retirement, and

$$
P_{t}=P_{t-1} U_{t}
$$

$F_{t}$ represents a non-stochastic component of non-financial income that determines the hump-shaped profile of "labor" (or non-financial) income over the life cycle in the absence of shocks. $V_{t}$ and $U_{t}$ are mutually independent iid random variables that are lognormally distributed with a mean of one. $U_{t}$ represents a shock component that has a permanent effect on labor income. $V_{t}$ represents a transitory shock component. Cocco et al. (2005) report estimations for (10), (11), as well as for the standard deviations of the logs of $V_{t}$ and $U_{t} \cdot{ }^{9}$ The calibrations here are based on their estimations for high school graduates.

During retirement, it is assumed that non-financial income is given by

$$
y_{t}=\delta y_{R-1} M_{t}
$$

The parameter $\delta$ represents a "replacement rate" and is set to .6. $M_{t} \in[\underline{M}, \bar{M}]$ represents a medical expenditure shock. The idea is that the medical expenditure shock reduces the amount of income that is available for normal spending. For simplicity, it is assumed that this shock is realized at age $R$ and is fully permanent. This means that $M_{R+i}=M_{R}$ for $1 \leq i \leq T-R$. Furthermore, it is assumed that $E_{t} M_{R}=1$ for $1 \leq t \leq R-1$.

\footnotetext{
${ }^{9}$ See their Tables $1,2,3$.
} 


\section{References}

Allen, T. W., Carroll, C. D., 2001. Individual learning about consumption. Macroeconomic Dynamics 5, 255-271.

Barberis, N., Thaler, R. H., 2003. A survey of behavioral finance. In: Constantinides, G. M., Harris, M., Stulz, R. M. (Eds.). Handbook of the Economics of Finance, Vol. 1, Amsterdam: Elsevier, 1053-1128.

Binswanger, J., 2009. Towards understanding life cycle saving of boundedly rational agents: A model with feasibility goals. Working paper, Tilburg University.

Bolton, P., Faure-Grimaud, A., 2009. Thinking ahead: The decision problem. Review of Economic Studies 76, 1205-1238.

Bowman, D., Minehart, D., Rabin, M., 1999. Loss aversion in a consumption-savings model. Journal of Economic Behavior and Organization 38, 155-178.

Campbell, J., 2006. Household finance. Journal of Finance 61, 1553-1604.

Carroll, C. D., 1997. Buffer-stock saving and the life cycle/permanent income hypothesis. Quarterly Journal of Economics 112, 1-55.

Choi, J., Laibson, D., Madrian, B. C., Metrick, A., 2009. Reinforcement learning and savings behavior. Journal of Finance 64, 2515-2534.

Cocco, J. F., Gomes, F. J., Maenhout, P. J., 2005. Consumption and portfolio choice over the life cycle. Review of Financial Studies 18, 491-533.

Friedman, M., 1953. The methodology of positive economics. In: Essays in Positive Economics, Chicago: Chicago University Press, 3-43.

Gabaix, X., Laibson, D., 2000. A boundedly rational decision algorithm. American Economic Review 90, Papers and Proceedings, 433-438.

Gabaix, X., Laibson, D., 2005. Bounded rationality and directed cognition. Working paper.

Gomes, F., Michaelides, A., 2003. Portfolio choice with internal habit formation: A life-cycle model with uninsurable labor income risk. Review of Economic Dynamics 6, 729-766. 
Gomes, F., Michaelides, A., 2005. Optimal life-cycle asset allocation: Understanding the empirical evidence. Journal of Finance 60, 869-904.

Laibson, D., 1997. Golden eggs and hyperbolic discounting. Quarterly Journal of Economics 112, 443-477.

Laibson, D., Repetto, A., Tobacman, J., 1998. Self-control and saving for retirement. Brookings Papers on Economic Activity, Vol. 1, 91-196.

Lettau, M., Uhlig, H., 1999. Rules of thumb versus dynamic programming. American Economic Review 89, 148-174.

Pemberton, J., 1993. Attainable non-optimality or unattainable optimality: A new approach to stochastic life cycle problems. The Economic Journal 103, 1-20.

Polkovnichenko, V., 2007. Life-cycle portfolio choice with additive habit formation preferences and uninsurable labor income risk. Review of Financial Studies 20, 83-124.

Shefrin, H. M., Thaler, R. H., 1988. The behavioral life-cycle hypothesis. Economic Inquiry 26, 609-643.

Thaler, R. H., 1994. Psychology and savings policies. American Economic Review 84, Papers and Proceedings, 186-192. 
Table 1: Equity shares over the life cycle (in percent)

\begin{tabular}{lcc}
\hline \hline Age & \multicolumn{2}{c}{ Equity share } \\
\cline { 2 - 3 } & Median & Mean \\
\hline$<30$ & 0 & 14.3 \\
$30-34$ & 0 & 22.3 \\
$35-39$ & 7.9 & 25.9 \\
$40-44$ & 22.5 & 30.7 \\
$45-49$ & 25.9 & 33.3 \\
$50-54$ & 30.0 & 34.4 \\
$55-59$ & 39.5 & 40.0 \\
$60-64$ & 34.0 & 36.1 \\
$65-69$ & 27.1 & 32.6 \\
\hline \hline Source: & Survey of Consumer \\
Finances 2004. &
\end{tabular}


Figure 1: Consumption and income

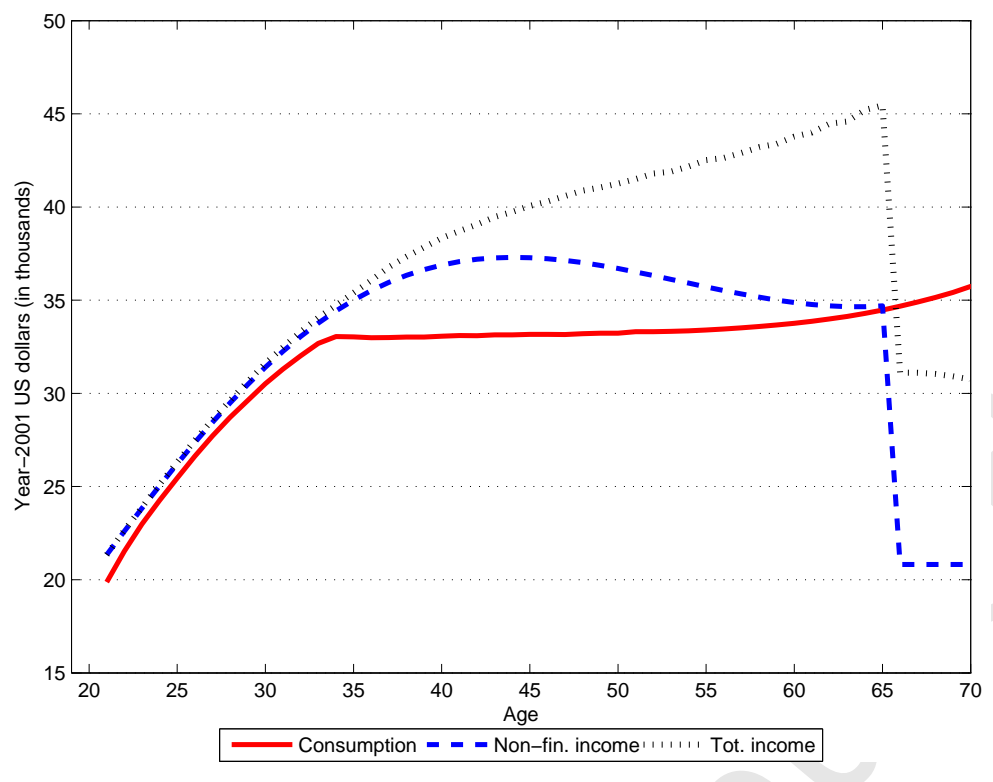

Figure 2: Equity shares

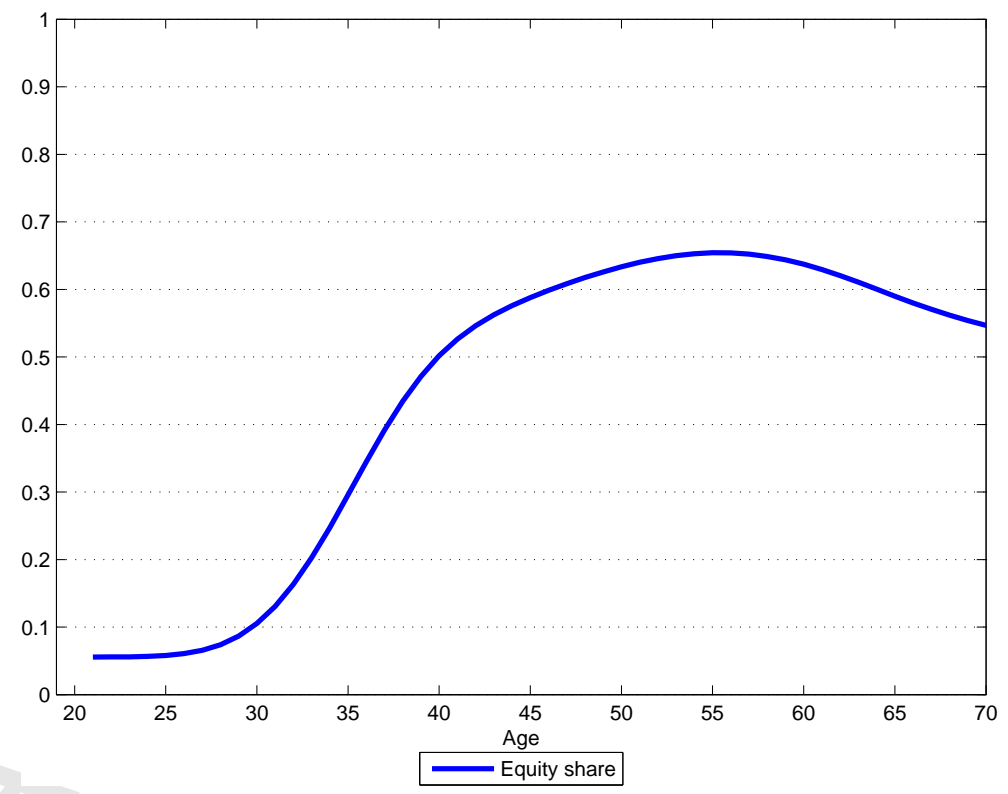

